\title{
Measurement Uncertainty Analysis of a Stitching Linear-Scan Method for the Evaluation of Roundness of Small Cylinders
}

\author{
Qiaolin Li, Yuki Shimizu*(D), Toshiki Saito, Hiraku Matsukuma and Wei Gao \\ Precision Nanometrology Laboratory, Department of Finemechanics, Tohoku University, Sendai 980-8579, Japan; \\ liqiaolin@nano.mech.tohoku.ac.jp (Q.L.); saito@nano.mech.tohoku.ac.jp (T.S.); \\ hiraku.matsukuma@nano.mech.tohoku.ac.jp (H.M.); gaowei@cc.mech.tohoku.ac.jp (W.G.) \\ * Correspondence: yuki.shimizu@nano.mech.tohoku.ac.jp; Tel.: +81-22-795-6950
}

Received: 22 June 2020; Accepted: 6 July 2020; Published: 10 July 2020

check for updates

\begin{abstract}
Influences of angular misalignments of a small cylinder on its roundness measurement by the method referred to as the stitching linear scan method are theoretically investigated. To compensate for the influences, a technique for measuring angular misalignments of a small cylinder by utilizing the linear-scan surface form stylus profilometer, which is employed for roundness measurement, is newly proposed. In addition, for roundness measurement, a holder unit capable of compensating for the angular misalignments of a small cylinder is developed, and the feasibility of the proposed technique is verified in experiments. Furthermore, a measurement uncertainty analysis of the stitching linear-scan method is carried out through numerical calculations based on a Monte Carlo method.
\end{abstract}

Keywords: precision metrology; form measurement; stitching linear-scan method; roundness measurement; measurement uncertainty; Monte Carlo method

\section{Introduction}

Roundness measurement is an important operation to assure the quality of cylinders [1]. Various sizes of small cylinders are employed in mechanical components; for example, a needle roller in a mechanical bearing [2]. Since the roundness of a needle roller affects the performance of the mechanical bearing in which the needle roller is integrated [3], it is necessary to evaluate the roundness of such a small cylinder with high precision [4].

In machine shops, a method with a dial gauge and a V-block is often employed in roundness measurement of a cylinder [5-7]. Meanwhile, the accuracy of roundness measurement in this method strongly depends on the skill of an operator, and it becomes much more difficult to carry out measurement with the decrease of the size of a workpiece. On the other hand, a roundness measuring instrument based on the rotary-scan method, in which a precision spindle and a displacement gauge are used, can carry out precise roundness measurement of a cylinder [8]. Compared with the V-block method described above, a roundness measuring instrument can reduce a deviation in roundness measurement induced by the operator. Meanwhile, even with a roundness measuring instrument, it becomes much more difficult to carry out measurement with the decrease of the size of a workpiece. Especially, the centering alignment of a small cylinder is a challenging task, and the influence of the eccentricity of a workpiece becomes much more significant with the decrease of the length and the diameter of a workpiece. Therefore, it has been difficult to carry out precision roundness measurement of a small cylinder having a diameter of less than a few millimeters [9].

In responding to the background described above, an alternative method referred to as the stitching linear-scan method has been proposed [10]. In the method, a series of arc-profiles around the 
circumference of a small cylinder is obtained by using a linear-scan surface form stylus profiler [11], which is often employed for measurement of a surface form/roughness [12], and the roundness profile is reconstructed by stitching the obtained arc-profiles. It has been revealed that the proposed method can carry out roundness measurement of a small cylinder having a diameter and a length of $1.5 \mathrm{~mm}$ and $5 \mathrm{~mm}$, respectively, which has been a challenging task using the conventional methods. Also, a good agreement can be found between the obtained roundness of a small cylinder with a diameter of $3 \mathrm{~mm}$ by the stitching linear-scan method and a conventional roundness measuring instrument. These results have demonstrated the feasibility of the proposed stitching linear-scan method. Meanwhile, in the proposed method, the angular misalignments of a small cylinder could affect the measurement uncertainty. Furthermore, an intensive analysis of the measurement uncertainty of the stitching linear-scan method has remained to be addressed.

To address the aforementioned issues, a new technique for the compensation of angular misalignments of a small cylinder in the stitching linear-scan method is proposed in this paper. The proposed technique is designed in such a way that a linear-scan surface form stylus profilometer for roundness measurement is utilized to detect angular misalignments of a small cylinder in a simple manner. An experimental setup capable of compensating for the angular misalignments is also developed, and the feasibility of the proposed technique is verified in experiments. Furthermore, numerical calculations are carried out based on a Monte Carlo method $[7,13]$ to estimate the measurement uncertainty of the stitching linear-scan method.

\section{Detection and Compensation of Angular Misalignments of a Small Cylinder in the Stitching Linear-Scan Method}

\subsection{Principle of the Stitching Linear-Scan Method}

Figure 1 shows a schematic of the setup for measurement of a small cylinder by the stitching linear-scan method [10]. The setup is composed of a linear-scan surface form stylus profilometer, a round magnet and a workpiece holder with a V-groove. A small cylinder to be measured is attached to the round magnet, on whose outer surface indexing marks with an angular interval of $45^{\circ}$ are prepared over $360^{\circ}$. Due to the magnetic force of the round magnet, a small cylinder can automatically be aligned to the center of the round magnet. It should be noted that there should be eccentricity of a small cylinder with respect to the round magnet. However, the influence of the eccentricity can be canceled during the following stitching operation; this is one of the advantages of employing the stitching linear-scan method [10]. The small cylinder with the round magnet is placed in the V-groove on the workpiece holder in such a way that the round magnet surface contacts the side of the work holder.

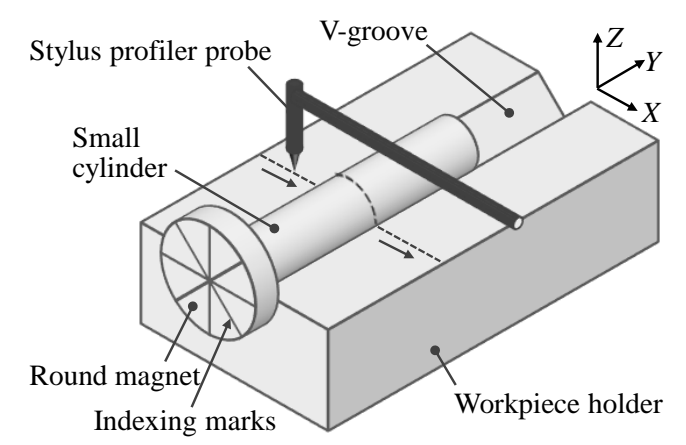

Figure 1. A schematic of the roundness measurement by the stitching linear-scan method.

Measurement of the circumferential profile of the small cylinder is carried out by repeating the $45^{\circ}$ rotation of the workpiece and the linear-scan of its arc-profiles, as shown in Figure 2. For the workpiece rotation, $n$ indexing marks ( $n$ is a small integer) prepared on the round magnet by a dividing head are utilized. By using the obtained eight arc-profiles of the workpiece, the workpiece profile over $360^{\circ} \mathrm{can}$ 
be obtained through the stitching process. From the obtained workpiece profile, evaluations of the roundness and diameter can be carried out. The cylinder-holding mechanism composed of the round magnet and the workpiece holder with the V-groove enables the linear-scan method to evaluate the whole length of a small cylinder, which cannot be achieved by the conventional roundness measuring instruments where a part of the cylinder in its axial direction should be held by a clamping mechanism. Furthermore, the precision positioning of a small cylinder along its axial direction can be achieved in a simple manner by utilizing the round magnet surface as the datum for the positioning.
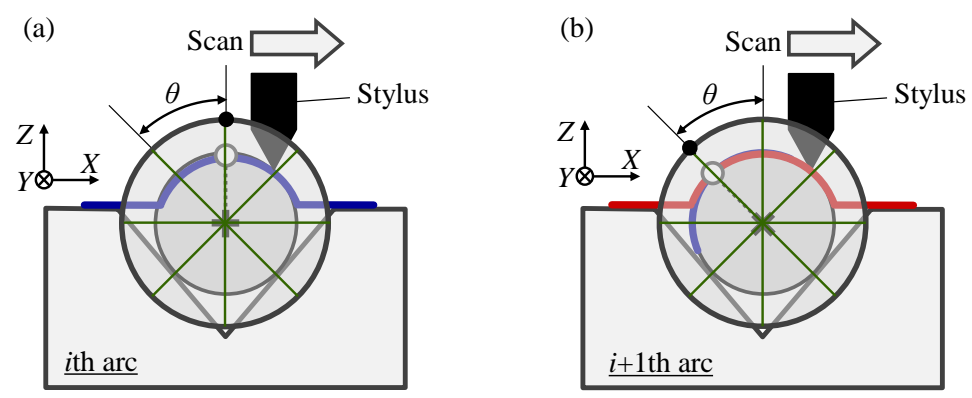

Figure 2. Measurement of the series of arc-profiles and the reconstruction of the circular profile of a workpiece: (a) measurement of the $i$ th arc profile; (b) measurement of the $i+1$ th arc profile.

The stitching process of the obtained eight arcs contains three-step calculations: the coordinate transformation, the radial stitching, and the circumferential stitching. Figure 3 shows a schematic of the stitching procedure. First we repeat the linear-scan and rotation of a small cylinder to obtain a series of arc profiles. After that, radial stitching is carried out for the obtained eight arcs. Each arc-profile datum of a small cylinder is in the Cartesian coordinate system $\left(x_{i, j}, z_{i, j}\right)$, where $i$ is the sampling number in each of the arcs, and $j(j=1,2, \ldots, 8)$ is the measurement order of the arc. By fitting a circle to each of the obtained arcs based on the least-squares method, the center coordinates and radius of the $j$ th arc $\left(x_{c, j}, z_{c, j}\right)$ and $r_{j}$, respectively, can be obtained. Then, the coordinates of the eight arcs are shifted based on the following equation to match the center coordinates of the arcs:

$$
\left(\begin{array}{l}
x^{\prime}{ }_{i, j} \\
z^{\prime}{ }_{i, j}
\end{array}\right)=\left(\begin{array}{c}
x_{i, j}-x_{c, j} \\
z_{i, j}-z_{c, j}
\end{array}\right)
$$

Furthermore, each arc profile is rotated by the following equation:

$$
\left(\begin{array}{l}
x^{\prime \prime}{ }_{i, j} \\
z^{\prime \prime}{ }_{i, j}
\end{array}\right)=\left(\begin{array}{cc}
\cos j \theta & \sin j \theta \\
-\sin j \theta & \cos j \theta
\end{array}\right)\left(\begin{array}{l}
x^{\prime}{ }_{i, j} \\
z^{\prime}{ }_{i, j}
\end{array}\right)
$$

It should be noted that each of the arcs has a different radius $r_{j}$. To stitch the arcs in the radial direction, a mean radius $\bar{R}$ is calculated by the following equation:

$$
\bar{R}=\frac{1}{8} \sum_{j=1}^{8} r_{j}
$$

By using the obtained $\bar{R}$, each sampling point in each of the arcs is adjusted by the following equation:

$$
r_{i, j}^{\prime}=r_{i, j}-r_{j}+\bar{R}
$$


where $r_{i, j}$ is the distance from the center of the arc to the point $\left(x_{i, j}^{\prime \prime}, z^{\prime \prime}{ }_{i, j}\right)$ in the $j$ th arc that can be calculated by the following equation:

$$
r_{i, j}=\sqrt{\left(x^{\prime \prime}{ }_{i, j}-x_{c, j}\right)^{2}+\left(z^{\prime \prime}{ }_{i, j}-z_{c, j}\right)^{2}}
$$

To minimize the deviation of each point from the fitting circle, $x_{c, j}, z_{c, j}$, and $r_{j}$ are determined by minimizing the residual sum of squares $S$ that can be calculated by the following equation [14]:

$$
S=\sum_{i=1}^{N}\left(\sqrt{\left(x^{\prime \prime}{ }_{i, j}-x_{c, j}\right)^{2}+\left(z_{i, j}^{\prime \prime}-z_{c, j}\right)^{2}}-r_{j}\right)^{2}
$$

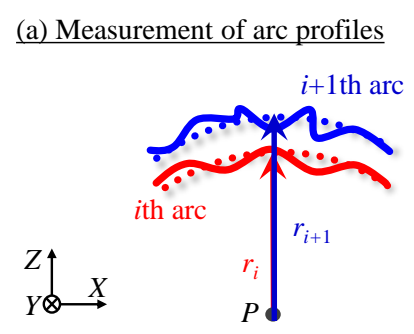

(c) Radial stitching

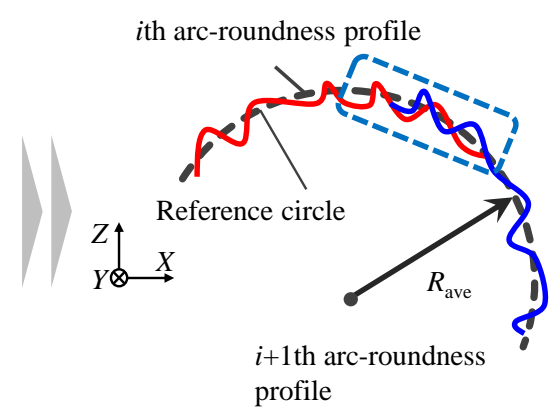

(b) Rotation of the arc profiles

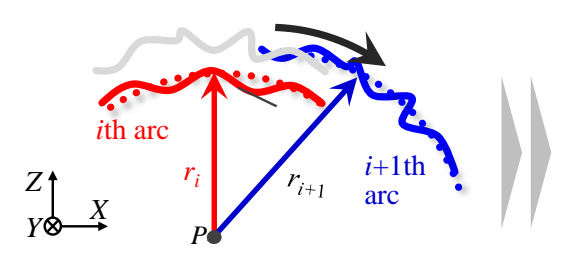

(d) Circumferential stitching

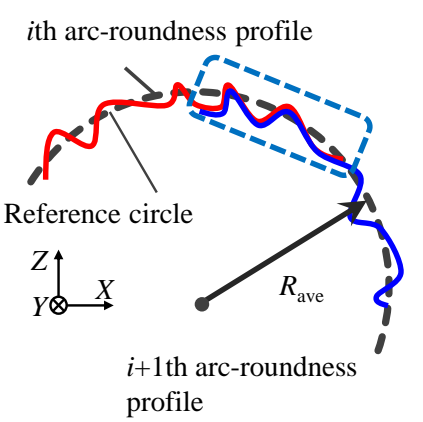

Figure 3. Stitching procedure for the reconstruction of the circular profile of a small cylinder: (a) measurement of arc profiles; (b) rotation of the obtained arcs; (c) radial stitching; (d) circumferential stitching.

As the next step of the stitching process, the circumferential stitching is carried out. The angular position $\theta_{i, j}$ of the $i$ th sampled points in the $j$ th arc profile can be calculated by the following equation:

$$
\theta_{i, j}=\tan ^{-1}\left(\frac{z^{\prime \prime}{ }_{i, j}}{x^{\prime \prime}{ }_{i, j}}\right)
$$

Since a small cylinder is positioned approximately by using the indexing marks prepared on the outer face of the round magnet, each of the arcs has a certain amount of misalignment in the circumferential direction. Denoting the compensating angle shift for the $j$ th arc as $\Delta \theta_{j}$, the angular position of the $i$ th point in the $j$ th arc can be calculated by the following equation:

$$
\theta^{\prime}{ }_{i, j}=\theta_{i, j}+\Delta \theta_{j}
$$

For the determination of $\Delta \theta_{j}$, a cross-correlation function $[15,16]$ can be employed. By stitching the neighboring arcs sequentially from the first arc to the last arc, circumferential stitching can be carried out. For the portions where the neighboring two arcs are overlapping, a mean value of the two radial coordinates can be employed. 
By using the profile of the workpiece obtained through the above procedures, the diameter $D$ and roundness of the workpiece can be evaluated. Meanwhile, the roundness can be evaluated by using the obtained profile data through processing it with the procedure defined by ISO [17]. For a small cylinder, the out-of-roundness $\Delta Z_{\theta}$ is evaluated based on the least square circle [18]. Denoting the mean distance of the sampling points from the center coordinates as $\bar{r}_{i}, \Delta Z_{\theta}$ can be evaluated by the following equation:

$$
\Delta Z_{\theta}=\left(\overline{r_{i}}\right)_{\operatorname{Max}}-\left(\overline{r_{i}}\right)_{\min }
$$

It should be noted that the eccentricity of the workpiece rotation will not affect the measurement in the proposed stitching linear-scan method [10]; this is one of the remarkable advantages compared with the conventional roundness measuring instruments, where the influence of the eccentricity becomes significant with the decrease of the diameter of a workpiece.

\subsection{Influences of the Angular Misalignments of a Roll Workpiece}

In the stitching linear-scan method, the angular misalignment $\theta_{X}$ of a small cylinder about the $X$-axis could affect the roundness measurement. Figure 4 shows a schematic of the influence of $\theta_{X}$ on the measured arc profile of a small cylinder. As can be seen in the figure, the radius of the observed arc becomes larger than the real one. Denoting the diameter of an ideal small cylinder as $D$, the $X$ - and $Z$-coordinates $\left(x_{i}, z_{i}\right)$ of the $i$ th sampled point satisfy the following equation:

$$
x_{i}^{2}+z_{i}^{2} \cos ^{2} \theta_{X}=D^{2} / 4
$$

(a)

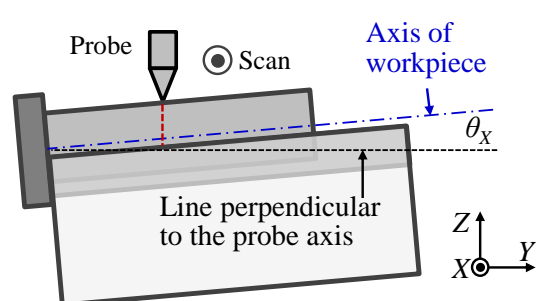

(b)

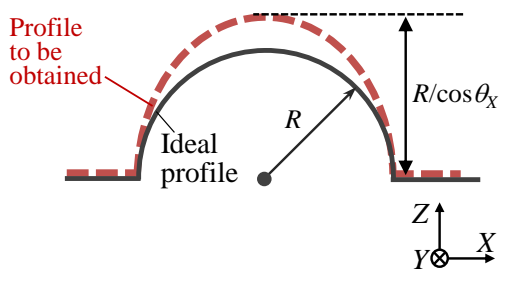

Figure 4. A schematic of the influence of angular misalignment $\theta_{X}$ on the arc-profile to be obtained in measurement: (a) definition of $\theta_{X} ;(\mathbf{b})$ error caused by $\theta_{X}$.

Denoting the $i$ th sampled point in the polar coordinate system as $\left(r_{i}, \theta_{i}\right)$, the above equation can be modified as follows:

$$
r_{i}\left(\theta_{i}\right)=\frac{D}{2}\left(\cos ^{2} \theta_{i}+\sin ^{2} \theta_{i} \cos ^{2} \theta_{X}\right)^{-\frac{1}{2}}
$$

The influence of the angular misalignment of the small cylinder about the $X$-axis $e_{\theta X}$ can thus be obtained by the following equation:

$$
e_{\theta X}\left(\theta_{i}\right)=\frac{D}{2}\left[1-\left(\cos ^{2} \theta_{i}+\sin ^{2} \theta_{i} \cos ^{2} \theta_{X}\right)^{-\frac{1}{2}}\right]
$$

Figure 5a shows the variation of $e_{\theta X}$ over an angular range from $\theta=42.5^{\circ}$ to $\theta=137.5^{\circ}$ under the condition of $D=1 \mathrm{~mm}$ and $\theta_{X}=1^{\circ}$. The error becomes maximum at the top of the arc (namely, $\theta=90^{\circ}$ ). Numerical calculations are extended to further investigate the influence of $\theta_{X}$. Figure $5 b$ shows the variation of the maximum $e_{\theta X}$ as the increase of $\theta_{X}$ under the condition of $\theta_{i}=42.5^{\circ}$. Calculations are carried out for the cases with different workpiece diameter $D$ ranging from $1 \mathrm{~mm}$ to $6 \mathrm{~mm}$. As can be seen in the figure, the influence of $\theta_{X}$ becomes larger with the increase of $D$. Meanwhile, the calculation results show that $e_{\theta X}$ can be suppressed to be less than $0.01 \mu \mathrm{m}$ by reducing $\theta_{X}$ to less than $0.3^{\circ}$ for a small cylinder with a diameter $D$ of less than $3 \mathrm{~mm}$, for which it is difficult to carry out roundness measurement by the conventional rotary-scan method. 
(a)

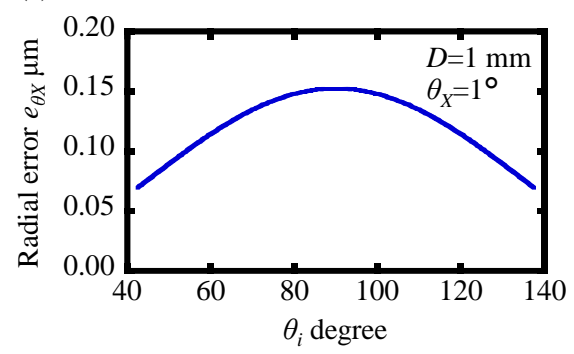

(b)

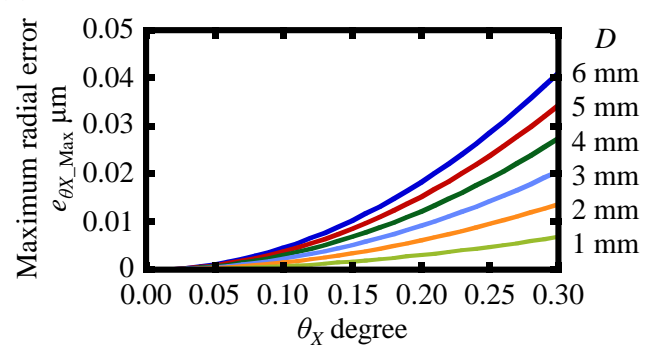

Figure 5. Radial error induced by the angular misalignment $\theta_{X}$ of a cylindrical workpiece: (a) radial error at each $\theta_{i} ;(\mathbf{b})$ variation of the maximum radial error due to the increase of $\theta_{X}$.

The angular misalignment $\theta_{Z}$ of a small cylinder about the Z-axis could also affect the roundness measurement. Figure 6 shows a schematic of the influence of $\theta_{Z}$ on the measured profile of a small cylinder. As can be seen in the figure, the radius of the observed arc becomes larger than the real one. The influence of the angular misalignment of the small cylinder about the Z-axis $e_{\theta Z}$ can be expressed by the following equation:

$$
e_{\theta z}\left(\theta_{i}\right)=\frac{D}{2}\left[1-\left(\cos ^{2} \theta_{i} \cos ^{2} \theta_{z}+\sin ^{2} \theta_{i}\right)^{-\frac{1}{2}}\right]
$$

(a)

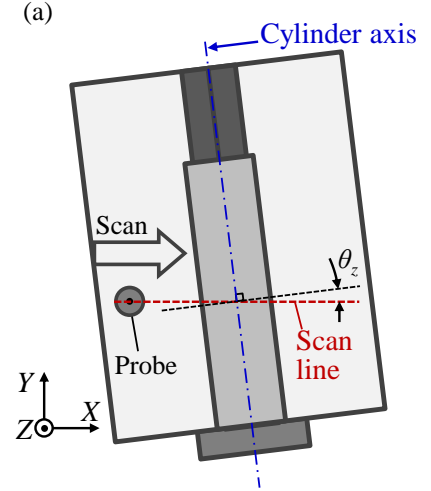

(b)

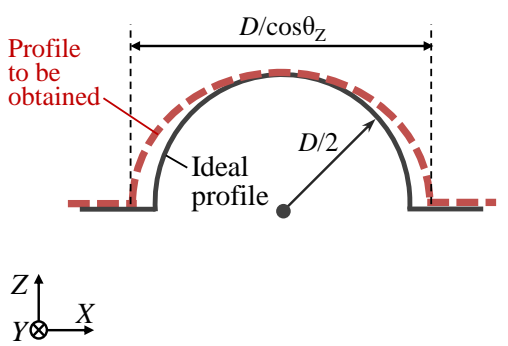

Figure 6. A schematic of the influence of angular misalignment $\theta_{Z}$ on the arc-profile to be obtained in measurement: (a) definition of $\theta_{Z}$; (b) error caused by $\theta_{Z}$.

In the same manner as $\theta_{X}$, the influence of $\theta_{Z}$ is also investigated through numerical calculations. Figure 7a shows the variation of $e_{\theta Z}$ under the condition of $D=1 \mathrm{~mm}$ and $\theta_{Z}=1^{\circ}$. The error becomes maximum at the edges of the arc (namely, $\theta_{i}=42.5^{\circ}$ and $137.5^{\circ}$ ). Figure $7 \mathrm{~b}$ shows the variation of the maximum $e_{\theta Z}$ as the increase of $\theta_{Z}$ under the condition of $\theta_{i}=42.5^{\circ}$. According to the calculation results, $e_{\theta Z}$ can be suppressed to be less than $0.01 \mu \mathrm{m}$ by reducing $\theta_{Z}$ to less than $0.2^{\circ}$ for a small cylinder with a diameter $D$ of less than $3 \mathrm{~mm}$. 
(a)

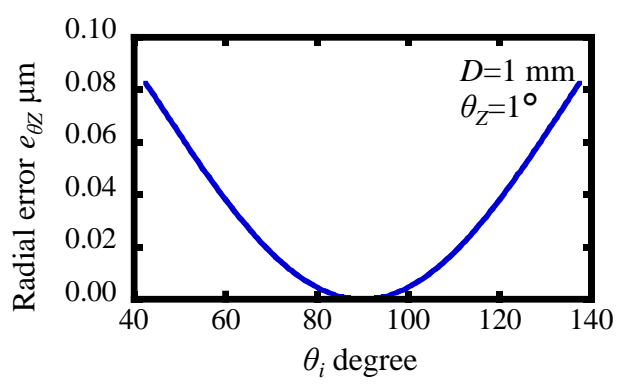

(b)

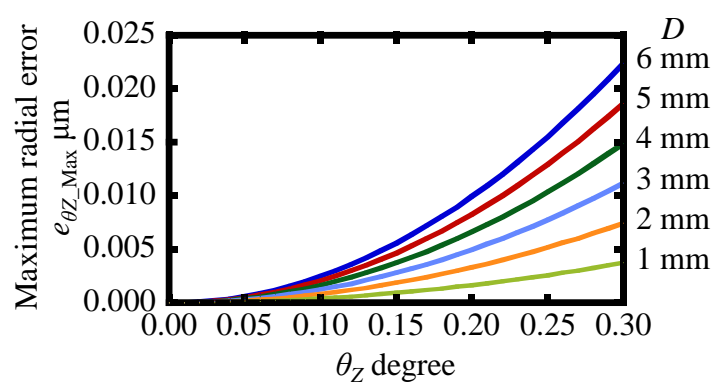

Figure 7. Radial error induced by the angular misalignment $\theta_{Z}$ of a cylindrical workpiece: (a) radial error at each $\theta_{i} ;(\mathbf{b})$ variation of the maximum radial error due to the increase of $\theta_{Z}$.

\subsection{A Technique to Evaluate Angular Misalignments of a Small Cylinder}

Regarding the results of numerical calculations described above, angular misalignments $\theta_{X}$ and $\theta_{Z}$ of a small cylinder need to be reduced as much as possible. It is thus necessary to establish a technique to detect $\theta_{X}$ and $\theta_{Z}$ in advance of the roundness measurement of a workpiece. However, regarding the small dimensions of a workpiece to be measured by the stitching linear-scan method, it is not so easy to apply conventional angle sensors such as an optical autocollimator [19] or a multi-axis laser autocollimator [20] for this purpose. To address the issue, attempts are made in this paper to utilize the linear scan surface form stylus profiler, which is employed for roundness measurement in the stitching linear-scan method, for the detection of $\theta_{X}$ and $\theta_{Z}$.

Now we consider a positioning system shown in Figure 8a composed of a tilt stage, a Z-rotary stage, and a workpiece holder. The tilt stage, the rotary stage, and the workpiece holder are stacked and assembled as a holder unit. Figure $8 \mathrm{~b}$ shows a photograph of the developed holder unit. The holder unit can be mounted on a linear stage.
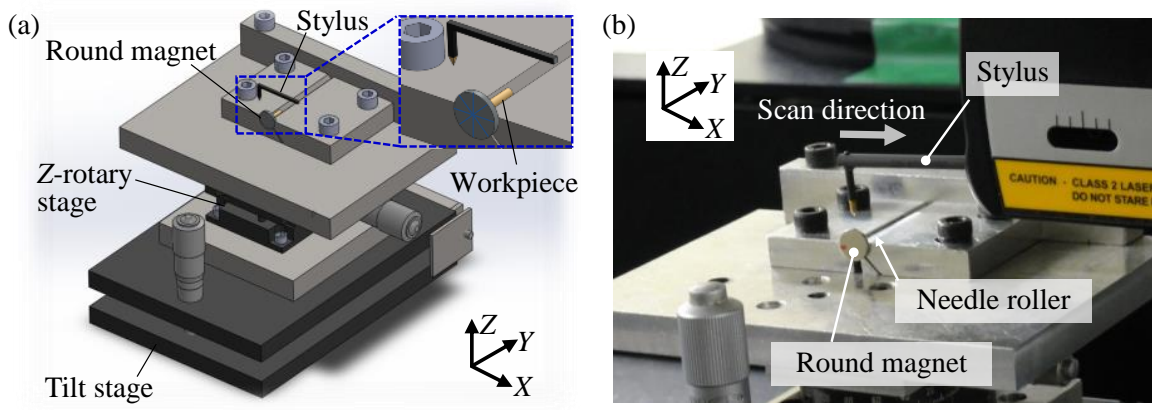

Figure 8. Setup for roundness measurement of a small cylinder with a surface profilometer: (a) a schematic of the holder unit; (b) a photograph of the holder unit with a surface profilometer.

In the proposed method, $\theta_{X}$ is detected through a two-step procedure. In the first step, the workpiece axis is aligned to be parallel with the $X$-axis as shown in Figure 9a. By scanning over the workpiece holder surface with the stylus, profile data $\left(x_{i \_ \text {hold }}, z_{i \_ \text {hold }}\right)$ of the workpiece holder surface can be obtained. The linear approximation of the obtained profile based on the least squares method provides the inclination angle $\theta_{\text {Scan_}_{-} Y}$ of the workpiece holder surface with respect to the datum surface of the surface form stylus profiler. Denoting the inclination angle of the top surface of the linear stage about the $Y$-axis with respect to the datum surface of the surface form stylus profiler as $\theta_{\text {Linear_}} \gamma$, and the inclination of the workpiece holder surface about the $Y$-axis with respect to the top surface of the linear stage as $\theta_{\text {Holder }}$, the following relationship should be satisfied:

$$
\theta_{\text {Scan_}} Y=\theta_{\text {Linear } \_} Y+\theta_{\text {Holder }}
$$


(a) Workpiece axis

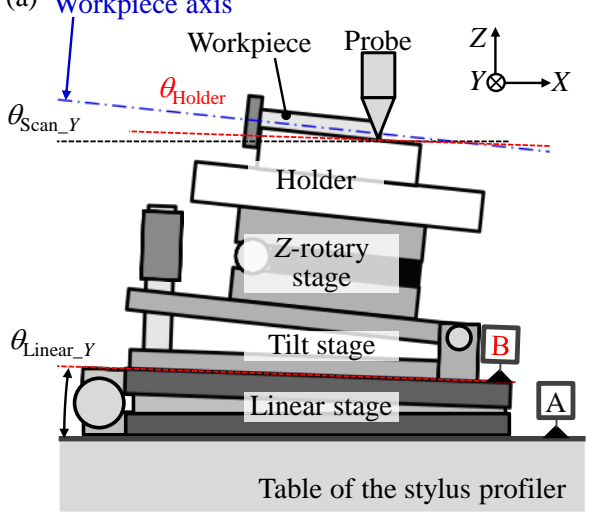

(b)

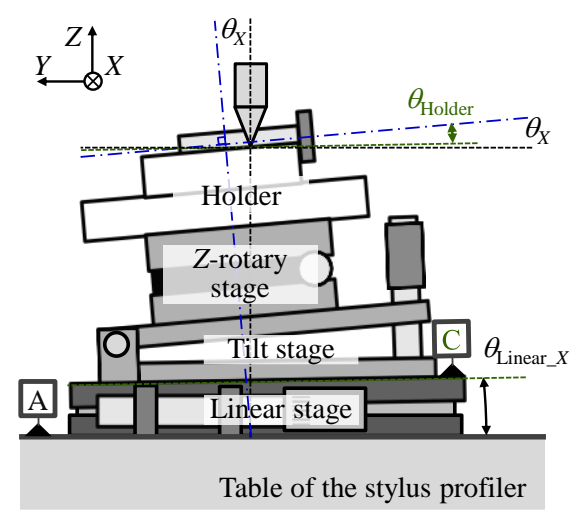

Figure 9. Evaluation of the angular misalignment $\theta_{X}$ of a small cylinder about the $X$-axis: (a) before the rotation of the holder unit about the Z-axis; (b) after $90^{\circ}$ rotation of the holder unit about the Z-axis.

In the second step, the holder unit is rotated $90^{\circ}$ about the Z-axis on the linear stage through the demounting and remounting operations, as shown in Figure $9 \mathrm{~b}$. It should be noted that $\theta_{\text {Holder }}$ does not change during the operations since the components in the holder unit are rotated together without separation. Denoting the inclination angle of the top surface of the linear stage about the $X$-axis with respect to the datum surface of the surface form stylus profiler as $\theta_{\text {Linear_X }}$, the angular position of the workpiece holder surface about the $X$-axis with respect to the datum surface of the surface form stylus profiler $\theta_{X}$ can be expressed by the following equation:

$$
\theta_{x}=\theta_{\text {Linear } \_} X+\theta_{\text {Holoder }}
$$

From Equations (14) and (15), the following equation can be obtained:

$$
\theta_{X}=\theta_{\text {Scan_}} Y+\theta_{\text {Linear_X }}-\theta_{\text {Linear_}} Y
$$

Since $\theta_{\text {Linear_X }}$ and $\theta_{\text {Linear_}} Y$ can be treated as the known parameters through scanning the top surface of the linear stage by the surface form stylus profiler in advance of the roundness measurement, $\theta_{X}$ can be evaluated by measuring $\theta_{\text {Scan_}} Y$ in the first step.

It should be noted that the parallelism of the cylinder/workpiece with respect to the $X$-axis was confirmed visually, and that there should exist a certain amount of angular misalignment of the workpiece about the $Z$-axis $\left(\varphi_{Z}\right)$, which could affect the measurement. According to the geometric relationship, the error $e_{\theta X}$ in measurement of $\theta_{X}$ due to $\varphi_{Z}$ can be expressed by the following equation:

$$
e_{\theta X}=\theta_{X}-\tan ^{-1}\left(\cos \varphi_{Z} \tan \theta_{X}\right)
$$

Under the condition of $\theta_{Z}=2.5^{\circ}$ and $\theta_{X}=0.1^{\circ}, e_{\theta X}$ becomes approximately $0.002^{\circ}$, and is small enough to be neglected. Meanwhile, the influence of the demounting and remounting operations of the holder unit in measurement of $\theta_{X}$ should be evaluated in experiments.

$\theta_{Z}$ can be evaluated by obtaining the arc profiles of a small cylinder at different $Y$-positions with enough intervals. Figure 10 shows a schematic of the evaluation of $\theta_{Z}$. First, we obtain the arc-profile of a workpiece at a $Y$-position of $y_{1}$, and detect the $X$-coordinate of the peak $\left(x_{\text {peak } 1}\right)$ in the obtained arc-profile through fitting a circle based on the least squares method. In the same manner, we detect the $X$-coordinate of the peak $\left(x_{\text {peak } 2}\right)$ in the arc-profile obtained at a $Y$-position of $y_{2}$. From the obtained peak coordinates, $\theta_{Z}$ can be calculated by the following equation:

$$
\theta_{z}=\tan \left(\frac{x_{\text {peak } 2}-x_{\text {peak } 1}}{y_{2}-y_{1}}\right)
$$


(a)

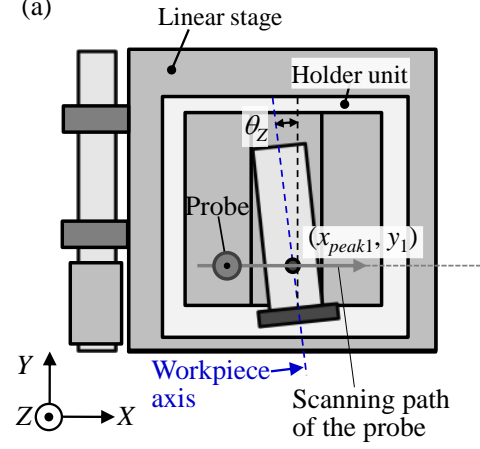

(b)

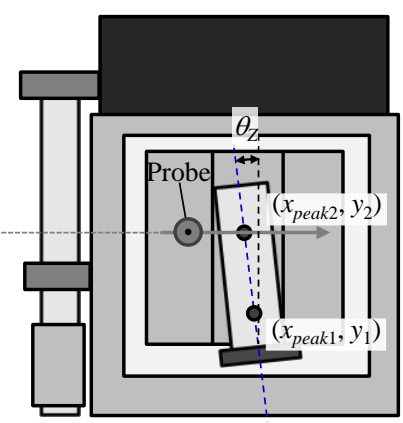

Figure 10. Evaluation of the angular misalignment $\theta_{Z}$ of a small cylindrical workpiece about the Z-axis:

(a) before shifting; (b) after shifting.

It should be noted that the angular misalignment of the workpiece about the $X$-axis $\left(\varphi_{X}\right)$ could affect the evaluation of $\theta_{Z}$. From the geometric relationship, the error $e_{\theta Z}$ in measurement of $\theta_{Z}$ due to $\varphi_{X}$ can be expressed by the following equation:

$$
e_{\theta Z}=\theta_{Z}-\tan ^{-1}\left(\cos \varphi_{X} \tan \theta_{Z}\right)
$$

In the proposed method, the evaluation of $\theta_{Z}$ is carried out after the measurement and alignment of $\theta_{X}$. On the assumption that $\theta_{X}$ is aligned to be less than $0.1^{\circ}, e_{\theta Z}$ becomes $2.66 \times 10^{-60}$ under the condition of $\theta_{Z}=0.1^{\circ}$; the influence of $\theta_{X}$ can thus be treated as small enough to be neglected.

\section{Experiments}

\subsection{Verification of the Evaluation Method of the Angular Misalignments of a Small Cylinder}

Experiments were carried out to verify the feasibility of the proposed technique for evaluation of the angular misalignments of the workpiece in roundness measurement of a small cylinder by the stitching linear-scan method. In the following verification experiments, a calibrated pin gauge with a diameter of $3.000 \mathrm{~mm}$ was employed as the measurement specimen. At first, basic characteristics of the proposed technique for the evaluation of $\theta_{X}$ were investigated in experiments. Figure 11 shows a schematic of the experimental setup. Experimental conditions are summarized in Table 1. The axis of a small cylinder was aligned to be parallel with the scanning direction of the surface form stylus profilometer ( $X$-axis in the figure). A commercial autocollimator was employed as the reference angle sensor for measurement of the angular displacement of the workpiece holder. A flat mirror was employed as the target for the commercial autocollimator and was mounted on the workpiece holder. The workpiece holder surface was scanned by the surface form stylus profilometer to obtain the inclination angle $\theta_{\text {Scan_}} Y$ of the workpiece holder surface with respect to the datum surface of the surface form stylus profiler.

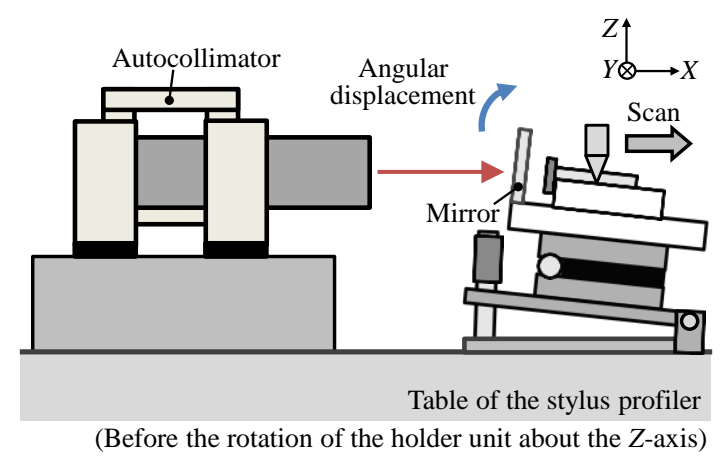

Figure 11. Setup for the verification of the method for measurement of $\theta_{X}$. 
Table 1. Parameters for scanning experiments.

\begin{tabular}{ccc}
\hline Items & Value & Unit \\
\hline Tip angle of a stylus & 60 & degree \\
Tip radius of a stylus & 2 & $\mu \mathrm{m}$ \\
Probe scanning speed & 0.1 & $\mathrm{~mm} / \mathrm{s}$ \\
Measuring force & 1.0 & $\mathrm{mN}$ \\
\hline
\end{tabular}

Figure 12a shows an example of the obtained surface profile of the workpiece holder. From the obtained profile data, the inclination angle $\theta_{\text {Scan_} \_}$was obtained through the linear fitting by the least-squares method. Ten repetitive trials, including the demounting and remounting operations of the holder unit at each trial, were made to evaluate the reproducibility. Figure $12 \mathrm{~b}$ shows the results. The standard deviation of $\theta_{\text {Scan_}} Y$ was evaluated to be $0.005^{\circ}$.

(a)

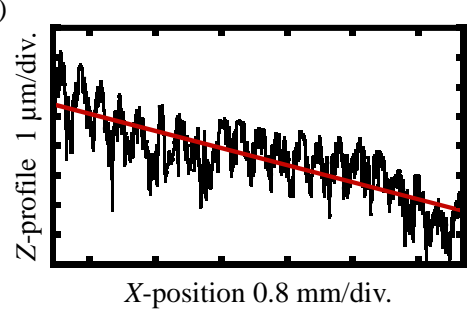

(b)

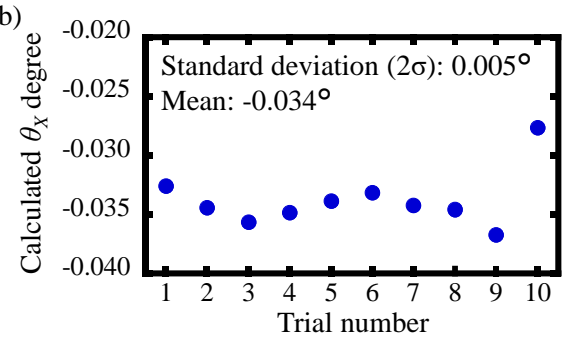

Figure 12. Evaluation of the $\theta_{X}$ and its reproducibility: (a) measured profile of the workpiece holder; (b) reproducibility of the evaluation of $\theta_{X}$.

Following the verification of the measurement reproducibility, an attempt was made to detect the angular displacement of the workpiece holder. By using the tilt stage, the workpiece holder was rotated about the $Y$-axis in steps of approximately $0.005^{\circ}$, and the angular displacement of the workpiece holder was evaluated by both the proposed method and the commercial autocollimator at each step. It should be noted that the scanning length of the stylus profilometer for evaluation of $\theta_{\text {Scan_}} Y$ was set to be $5 \mathrm{~mm}$. Figure 13 shows the results. As can be seen in the figure, the maximum deviation of the detected $\theta_{\text {Scan_}} \gamma$ from the reading of the autocollimator was less than $\pm 0.002^{\circ}$. These experimental results demonstrated the feasibility of the proposed technique of measuring $\theta_{\text {Scan_}} \gamma$.

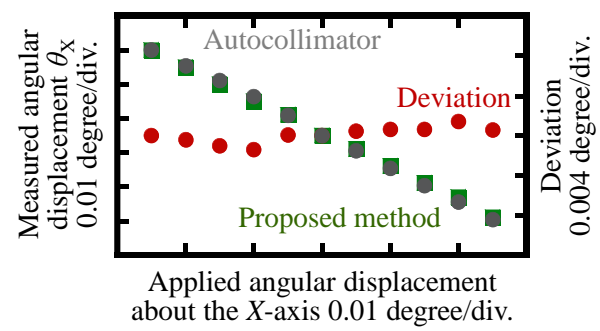

Figure 13. The angular displacement of the workpiece about the $X$-axis measured by the proposed technique.

Experiments were also carried out to evaluate the basic characteristics of the proposed technique for the evaluation of $\theta_{Z}$. Figure 14 shows a schematic of the experimental setup. The pin gauge was first attached to a round magnet and was then placed on the V-groove in the workpiece holder. The axis of the workpiece was aligned to be parallel with the $Y$-axis so that its arc-profiles could be measured by the stylus profilometer. 


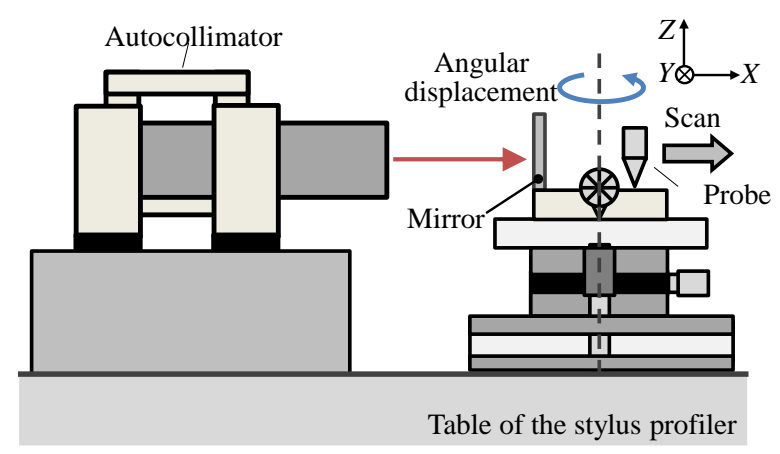

Figure 14. Setup for the verification of the technique for measurement of $\theta_{Z}$.

By using the setup, the arc-profile of the workpiece was first obtained at a $Y$-position of $y_{1}$. After that, the workpiece was made to travel along the $Y$-direction together with the holder unit by using the linear stage. After that, another arc-profile was obtained at a $Y$-position of $y_{2}\left(=y_{1}+\Delta y\right)$. Figure $15 \mathrm{a}$ shows an example of the arc-profile obtained during the experiments. For each of the obtained arc-profiles, a circle was fitted based on the least-squares method to obtain the peak coordinate as shown in Figure 15b. After that, by using the obtained data, $\theta_{Z}$ was evaluated based on Equation (18). In the experiments, $\Delta y$ was set to $4 \mathrm{~mm}$. Five repetitive trials were made by following the above procedure without the demounting and remounting operations of the holder unit at each trial, and the standard deviation of the measurement of $\theta_{Z}$ was evaluated to be $0.006^{\circ}$, as shown in Figure $15 \mathrm{c}$.
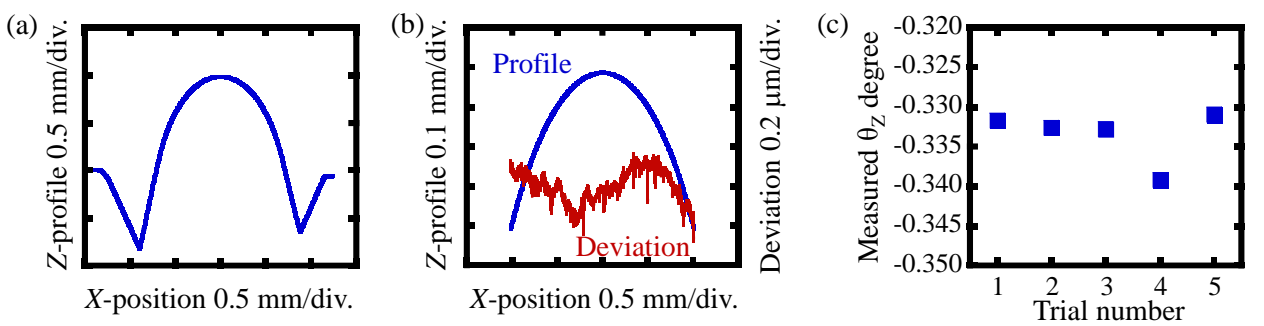

Figure 15. Evaluation of the $\theta_{Z}$ and its repeatability: (a) measured profile (b) arc-profile cut from the profile in (a) and its deviation from a fitting circle; (c) repeatability of $\theta_{Z}$ measurement.

Following the verification of the measurement repeatability, an attempt was made to detect the angular displacement $\theta_{Z}$ of the pin gauge. By using the rotary stage, the workpiece was rotated about the $Z$-axis in steps of approximately $0.023^{\circ}$, corresponding to the resolution of the rotary stage. The angular displacement of the workpiece was evaluated by both the proposed technique and the commercial autocollimator at each step. Figure 16 shows the results. As can be seen in the figure, the maximum deviation of the detected $\theta_{Z}$ from the reading of the autocollimator was less than $0.01^{\circ}$. These experimental results demonstrate the feasibility of the proposed technique for measurement of $\theta_{Z}$. By using the obtained $\theta_{X}$ and $\theta_{Z}$, the angular misalignment of the workpiece can thus be compensated.

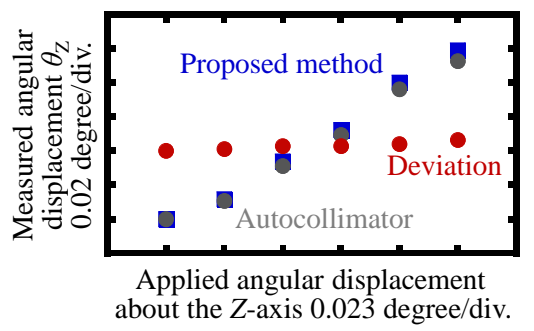

Figure 16. The angular displacement of the workpiece about the Z-axis measured by the proposed method. 


\subsection{Evaluation of the Roundness Profile of a Small Cylinder with a Diameter of $3 \mathrm{~mm}$}

After the compensation of the angular misalignments $\theta_{X}$ and $\theta_{Z}$ of the workpiece by the proposed technique, roundness measurement was carried out. Figure 17a shows the series of arc-profiles obtained in measurements. Figure 17b shows the profile reconstructed from the obtained arc-profiles before the stitching process. To evaluate the effectiveness of the compensation of the angular misalignments, experiments were carried out for the cases of $\theta_{Z}=0.004^{\circ}$ and $\theta_{Z}=0.951^{\circ}$.

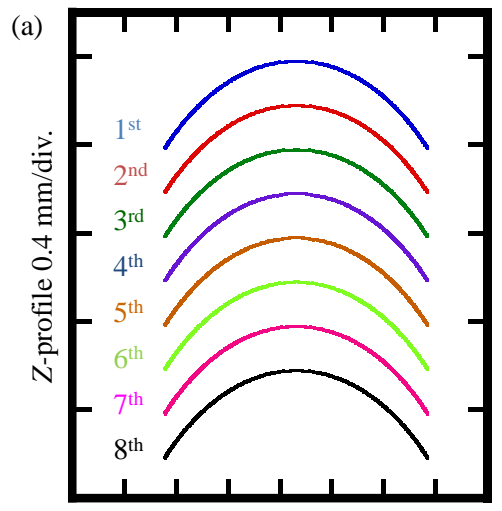

$X$-position $0.4 \mathrm{~mm} / \mathrm{div}$.

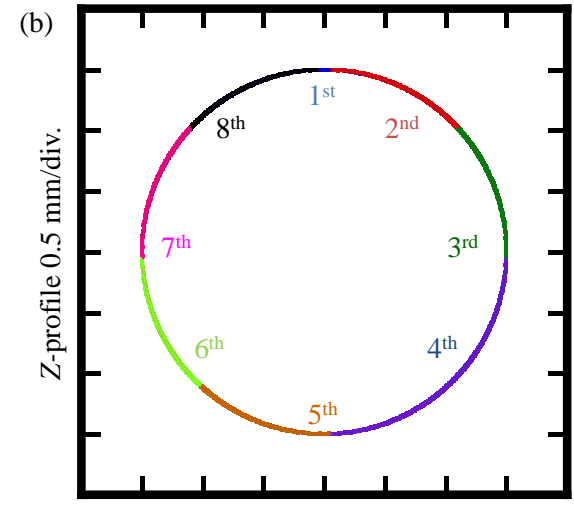

$X$-position $0.5 \mathrm{~mm} / \mathrm{div}$.

Figure 17. Profiles of a pin gauge with a diameter of $3 \mathrm{~mm}$ measured by the stitching linear-scan method: (a) measured arc profiles; (b) the arc profiles in (a) after the rotation of each arc.

Figure 18 shows the roundness profiles of the workpiece before and after the stitching process and after the filtering process. The results are summarized in Table 2. As can be seen in the figure and table, the angular misalignment $\theta_{Z}$ was found to affect the result of roundness measurement in the stitching linear-scan method.

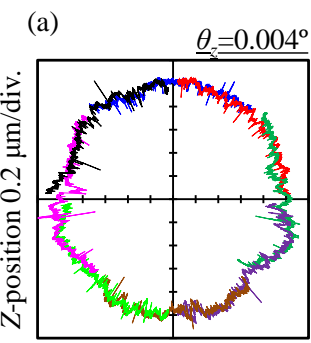

$X$-position $0.2 \mu \mathrm{m} / \mathrm{div}$.

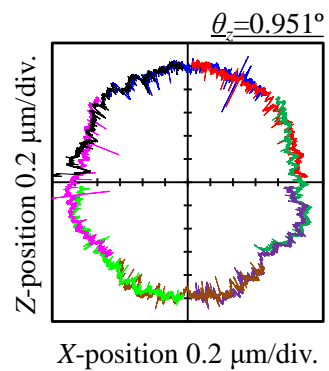

(b)
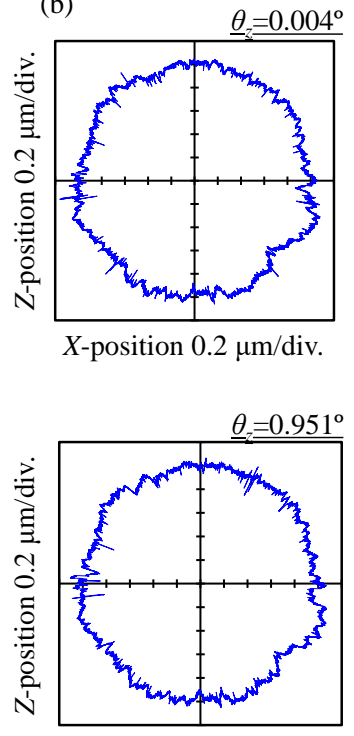

$X$-position $0.2 \mu \mathrm{m} /$ div.
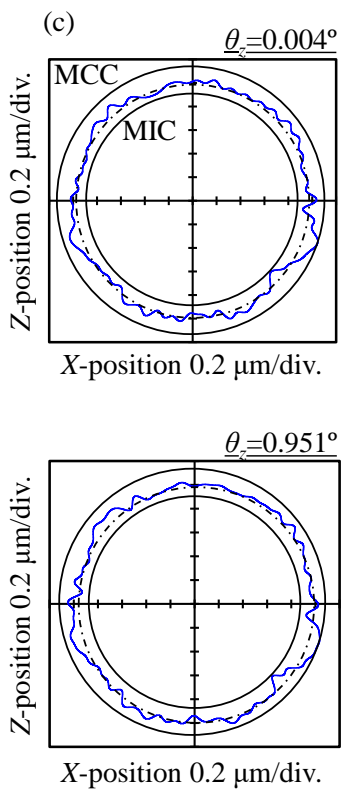

Figure 18. Evaluation of the roundness by the obtained arc profiles: (a) roundness profiles after radial stitching; (b) roundness profiles after circumferential stitching; (c) roundness profiles after filtering. 
Table 2. Radius and roundness.

\begin{tabular}{cccc}
\hline Angular Misalignment of the Pin Gage $\boldsymbol{\theta}_{\boldsymbol{z}}$ & $\mathbf{0 . 0 0 4}^{\circ}$ & $\mathbf{0 . 9 5 1}^{\circ}$ \\
\hline \multirow{2}{*}{ Diameter } & Mean value & $2.99963 \mathrm{~mm}$ & $2.99898 \mathrm{~mm}$ \\
& Standard deviation & $0.10 \mu \mathrm{m}$ & $0.13 \mu \mathrm{m}$ \\
\hline \multirow{2}{*}{ Roundness } & Mean value & $0.21 \mu \mathrm{m}$ & $0.19 \mu \mathrm{m}$ \\
& Standard deviation & $0.03 \mu \mathrm{m}$ & $0.01 \mu \mathrm{m}$ \\
\hline
\end{tabular}

\section{Measurement Uncertainty Analysis of the Stitching Linear Scan Method}

Theoretical investigation of the measurement uncertainty of the stitching linear-scan method was carried out. In the method, several arc-profiles were first measured in the Cartesian coordinate system $\left(x_{i}, z_{i}\right)$. After that, the obtained data of the arc-profiles were converted into the polar coordinate system $\left(r_{i}, \theta_{i}\right)$ to reconstruct the workpiece profile through the stitching process. Finally, the roundness of the workpiece was evaluated by the reconstructed profile. Since the above procedure contained a fitting process based on the least-squares method, numerical calculations based on the Monte Carlo method were carried out for the estimation of the measurement uncertainty. Since the stitching linear-scan method was developed for roundness measurement of a small cylinder having a radius of a few millimeters, following the previous work by the authors [10], a series of arc-profiles obtained by measuring a needle roller with a diameter of $1.5 \mathrm{~mm}$ was employed for the following numerical calculations.

First, the uncertainty of the radius of each sampling point was estimated. On the assumption that the workpiece had a perfect cylindrical profile, the radius $r_{i}$ at the $i$ th sampled point $\left(x_{i}, \mathrm{z}_{i}\right)$ could be expressed by the following equation:

$$
r_{i}=\sqrt{x_{i}^{2}+z_{i}^{2}}
$$

The standard uncertainty of $r_{i}\left(u_{r i}\right)$ can thus be obtained by the following equation:

$$
u_{r i}=\sqrt{\left(\frac{\partial R_{i}}{\partial x_{i}}\right)^{2} u_{x i}{ }^{2}+\left(\frac{\partial R_{i}}{\partial z_{i}}\right)^{2} u_{z i}{ }^{2}}=\sqrt{\frac{x_{i}^{2}}{x_{i}^{2}+z_{i}^{2}} u_{x i}{ }^{2}+\frac{z_{i}^{2}}{x_{i}^{2}+z_{i}^{2}} u_{z i}{ }^{2}}
$$

For the evaluation of $u_{r i}$, the uncertainties of $z_{i}$ and $x_{i}$ need to be obtained. Table 3 summarizes the sources of uncertainty contributing to the uncertainty of $z_{i}$. The stylus profilometer employed in the series of experiments was calibrated by using a master sphere artifact in advance of the roundness measurements. According to the specification sheet of the stylus profilometer [11], the uncertainty of the calibration is within $\pm 200 \mathrm{~nm}$. It should be noted that the contribution due to the drift between calibrations is included in this value. Assuming the rectangular probability distribution with a divisor of $\sqrt{3}$, the standard uncertainty of the calibration $\left(u_{\text {Cal }}\right)$ was evaluated to be $115.47 \mathrm{~nm}$. It should be noted that $u_{\text {Cal }}$ contains the influences of the straightness of the linear scan axis, the form errors of the master sphere artifact, and the stylus tip. Also, the uncertainty associated with the resolution of the Z-reading $\left(u_{\text {res_z }}\right)$ was evaluated to be $0.92 \mathrm{~nm}$, with regard to the value $(3.2 \mathrm{~nm})$ shown in the specification sheet of the stylus profiler. Furthermore, the contribution of repeatability of the Z-reading $\left(u_{\text {rep_z }} z\right)$ was estimated from a standard deviation in ten repetitive arc-profile measurements $(67.66 \mathrm{~nm})$. A standard uncertainty of $z_{i}\left(u_{z i}\right)$ was thus evaluated to be $133.84 \mathrm{~nm}$. 
Table 3. Uncertainty of the Z-coordinate of each sampled point.

\begin{tabular}{|c|c|c|c|c|}
\hline Sources of Uncertainty & Symbol & Type & $\begin{array}{l}\text { Probability } \\
\text { Distribution }\end{array}$ & $\begin{array}{c}\text { Standard } \\
\text { Uncertainty }\end{array}$ \\
\hline $\begin{array}{l}\text { Calibration of the probe (including } \\
\text { the contribution due to the drift } \\
\text { between calibrations) }\end{array}$ & $u_{\mathrm{Cal}}$ & B & Rectangular & $115.47 \mathrm{~nm}$ \\
\hline Resolution of probe & $u_{\text {Res } \_z}$ & B & Rectangular & $0.92 \mathrm{~nm}$ \\
\hline Repeatability & $u_{\text {Rep }}$ & A & Gaussian & $67.66 \mathrm{~nm}$ \\
\hline Standard uncertainty of $z_{i}$ & $u_{z i}$ & & & $133.84 \mathrm{~nm}$ \\
\hline
\end{tabular}

The uncertainty of $x_{i}$ was also estimated. Table 4 summarizes the sources of uncertainty contributing to the uncertainty of $x_{i}$. According to the manufacturer of the stylus profilometer, the standard uncertainty of the reading of $X$-position $\left(u_{\text {Read } \_x}\right)$ is $150 \mathrm{~nm}$. It should be noted that this value contains the contributions from the repeatability, the drift, and so on. Also, the contribution of the resolution of the $x$-reading $\left(u_{\text {Res_ } x}\right)$ was evaluated to be $36.08 \mathrm{~nm}$, with regard to the value $(125 \mathrm{~nm})$ shown in the specification sheet of the stylus profiler and the rectangular probability distribution with a divisor of $2 \sqrt{3}$. Meanwhile, for the evaluation of $u_{x i}$, influences of the angular misalignments of a workpiece about the $Z$ - and $X$-axes need to be considered. Denoting the angular misalignment of a workpiece about the $Z$-axis as $\theta_{Z}$, the resultant error in the $X$-coordinate of each sampled point $\Delta x_{i \_} \theta Z$ can be expressed by the following equation from the geometric relationship:

$$
\Delta x_{i_{-} \theta Z}=R \sin (\varphi / 2) \cdot\left[\left(1 / \cos \theta_{Z}\right)-1\right]
$$

Table 4. Uncertainty of the X-coordinate of each sampled point.

\begin{tabular}{|c|c|c|c|c|}
\hline Sources of Uncertainty & Symbol & Type & $\begin{array}{l}\text { Probability } \\
\text { Distribution }\end{array}$ & $\begin{array}{l}\text { Standard } \\
\text { Uncertainty }\end{array}$ \\
\hline $\begin{array}{l}\text { Reading of } x \text { (including the } \\
\text { contributions from the repeatability, } \\
\text { the drift and so on) }\end{array}$ & $u_{\text {read_x }} x$ & B & Gaussian & $150.00 \mathrm{~nm}$ \\
\hline Resolution of $x$-reading & $u_{\text {Res } \_x}$ & B & Rectangular & $36.08 \mathrm{~nm}$ \\
\hline Angular misalignment $\theta_{Z}$ & $u_{\theta Z}$ & B & Rectangular & $0.89 \mathrm{~nm}$ \\
\hline Angular misalignment $\theta_{X}$ & $u_{\theta X}$ & B & Rectangular & $0.89 \mathrm{~nm}$ \\
\hline Standard uncertainty of $x_{i}$ & $u_{x i}$ & & & $154.28 \mathrm{~nm}$ \\
\hline
\end{tabular}

The contribution of $\theta_{Z}\left(u_{\theta Z}\right)$ can thus be evaluated by the following equation:

$$
u_{\theta Z}=\sqrt{\left(\frac{\partial \Delta x_{i} \theta Z}{\partial \theta_{Z}}\right)^{2} u^{2}\left(\theta_{Z}\right)}=\sqrt{\left[R \sin \left(\frac{\varphi}{2}\right) \sin \theta_{Z} / \cos ^{2} \theta_{Z}\right]^{2} \cdot u^{2}\left(\theta_{Z}\right)}
$$

On the assumption that $\theta_{Z}$ was within $\pm 0.1^{\circ}$, by employing a mean radius $\bar{R}$ of $1.5 \mathrm{~mm}$ after the stitching process, $u_{\theta Z}$ was evaluated to be $0.89 \mathrm{~nm}$. The contribution of the angular misalignment of a workpiece about the $X$-axis $\theta_{X}$ was also estimated. According to the geometric relationship, the error in the $X$-coordinate due to $\theta_{X}\left(\Delta x_{i} \theta X\right)$ can be expressed by the following equation:

$$
\Delta x_{i_{-} \theta X}=r_{i} \sin (\varphi / 2) \cdot\left[1-\cos \theta_{X}\right]
$$

On the assumption that $\theta_{X}$ was within $\pm 0.1^{\circ}$, by employing a mean radius $\bar{R}$ of $1.5 \mathrm{~mm}$ after the stitching process, $u_{\theta X}$ was evaluated to be $0.89 \mathrm{~nm}$ in the same manner as $u_{\theta Z}$. By combining these contributions, the standard uncertainty of $x_{i}\left(u_{x i}\right)$ was evaluated to be $154.28 \mathrm{~nm}$. Compensations of the angular misalignment of a workpiece are effective in reducing the standard uncertainties of $x_{i}$ and 
$z_{i}$, and the contributions from the specifications of the stylus profiler were found to be dominant in these uncertainties.

By combining these contributions based on Equation (21), $u_{r i}$ was evaluated to be $178.75 \mathrm{~nm}$, as summarized in Table 5 . It should be noted that the sensitivity coefficients $\left(\partial r_{i} / \partial x_{i}\right)$ and $\left(\partial r_{i} / \partial z_{i}\right)$ were treated to have maximum values $\left(\partial r_{i} / \partial x_{i}\right)=\sin (\varphi / 2)=0.67559$ and $\left(\partial r_{i} / \partial z_{i}\right)=1$, respectively, to avoid underestimation.

Table 5. Uncertainty of the radius of each sampled point.

\begin{tabular}{|c|c|c|c|c|}
\hline Sources of Uncertainty & Symbol & Value & $\begin{array}{l}\text { Sensitivity } \\
\text { Coefficient }\end{array}$ & $\begin{array}{c}\text { Standard } \\
\text { Uncertainty }\end{array}$ \\
\hline Standard uncertainty of $z$ & $u_{z i}$ & $133.84 \mathrm{~nm}$ & 0.67559 & $90.42 \mathrm{~nm}$ \\
\hline Standard uncertainty of $x$ & $u_{x i}$ & $154.28 \mathrm{~nm}$ & 1 & $154.28 \mathrm{~nm}$ \\
\hline \multicolumn{3}{|c|}{ Standard uncertainty of $r_{i}$} & $u_{r i}$ & $178.75 \mathrm{~nm}$ \\
\hline
\end{tabular}

A standard uncertainty of $\theta_{i}\left(u_{\theta i}\right)$ was also evaluated. Since $\theta_{i}$ can be calculated as $\theta_{i}=\arctan \left(z_{i} / x_{i}\right)$, $u_{\theta i}$ can be calculated by the following equation:

$$
u_{\theta i}=\sqrt{\left(\frac{\partial \theta_{i}}{\partial x_{i}}\right)^{2} u_{x i}{ }^{2}+\left(\frac{\partial \theta_{i}}{\partial z_{i}}\right)^{2} u_{z i}{ }^{2}}=\sqrt{\left\{\frac{z_{i}}{x_{i}{ }^{2}+z_{i}{ }^{2}}\right\}^{2} u_{x i}{ }^{2}+\left\{\frac{x_{i}}{x_{i}{ }^{2}+z_{i}{ }^{2}}\right\}^{2} u_{z i}{ }^{2}}
$$

The sensitivity coefficients $\left(\partial \theta_{i} / \partial x_{i}\right)$ and $\left(\partial \theta_{i} / \partial z_{i}\right)$ were calculated as $\left(\partial \theta_{i} / \partial x_{i}\right)=1 / R$ and $\left(\partial \theta_{i} / \partial z_{i}\right)=(1 / R) \sin (\varphi / 2)$, respectively, to avoid underestimation. As a result, $u_{\theta i}$ was evaluated to be $u_{\theta i}=1.1 \times 10^{-4} \mathrm{rad}(0.0065$ degree $)$, as summarized in Table 6 .

Table 6. Uncertainty of the angular position of each sampled point.

\begin{tabular}{ccccc}
\hline Uncertainty Sources & Symbol & Value & $\begin{array}{c}\text { Sensitivity } \\
\text { Coefficient }\end{array}$ & $\begin{array}{c}\text { Standard } \\
\text { Uncertainty }\end{array}$ \\
\hline Standard uncertainty of $z_{i}$ & $u_{z i}$ & $133.84 \mathrm{~nm}$ & $666.67 \mathrm{rad} / \mathrm{mm}$ & $0.0000892 \mathrm{rad}$ \\
Standard uncertainty of $x_{i}$ & $u_{x i}$ & $154.28 \mathrm{~nm}$ & $450.39 \mathrm{rad} / \mathrm{mm}$ & $0.0000695 \mathrm{rad}$ \\
\hline Standard uncertainty of $\theta_{i}$ & $u_{\theta i}$ & \multicolumn{3}{c}{$0.00011 \mathrm{rad}(0.0065 \mathrm{deg})}$. \\
\hline
\end{tabular}

By using the obtained $u_{r i}$ and $u_{\theta i}$, numerical calculations were carried out based on the Monte Carlo method. The procedure of the numerical calculations was as follows:

Step 1: Prepare the data of the series of arc profiles in the polar coordinate system by using the circumferential profile of a small cylinder obtained in experiments after filtering (50 URP).

Step 2: Apply a random value in a Gaussian distribution with a standard deviation of $u_{\theta}$ to the $\theta$-coordinate of each point.

Step 3: Apply a random value in a Gaussian distribution with a standard deviation of $u_{R}$ to the $R$-coordinate of each point.

Step 4: Convert the arc-profile data into the Cartesian coordinate system.

Step 5: Carry out the stitching process in the same manner as the experiments.

Figure 19 shows the results of numerical calculations. A trial number of $1 \times 10^{5}$ was used for sufficient numerical stability of the output parameters. Expanded uncertainties of diameter and roundness were thus evaluated to be $0.032 \mu \mathrm{m}$ and $0.024 \mu \mathrm{m}(k=2,95 \%$ confidence), respectively. 


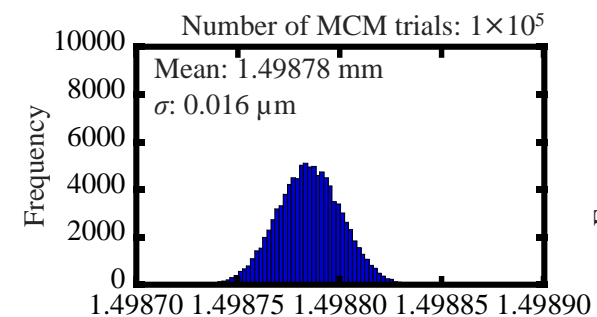

(a) Diameter $D \mathrm{~mm}$

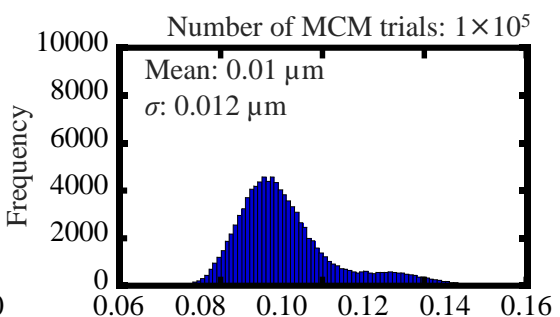

(b) Roundness $\mu \mathrm{m}$

Figure 19. Uncertainties of a radius and roundness estimated by numerical calculations based on the Monte Carlo method: (a) radius; (b) roundness.

\section{Conclusions}

A technique to compensate for angular misalignments of a small cylinder in the stitching linear-scan method has been proposed. In the proposed technique, the angular misalignment of a small cylinder about the $X$-axis was evaluated by measuring the inclination angle of a workpiece holder surface by using the stylus profiler, while the one about the Z-axis was evaluated by using the data of two arc-profiles obtained at different axial positions of the workpiece. Experimental results demonstrated that the proposed method can evaluate the angular misalignments with a repeatability/reproducibility of better than $0.01^{\circ}$, which is enough to suppress the measurement uncertainty of a workpiece diameter as well as roundness. Measurement of a pin gauge having a diameter and a length of $3 \mathrm{~mm}$ and $5 \mathrm{~mm}$, respectively, was carried out, and the feasibility of the proposed technique was verified. Furthermore, measurement uncertainty analysis was carried out through numerical calculations based on a Monte Carlo method to theoretically verify the feasibility of the stitching linear-scan method. Expanded uncertainties for workpiece diameter measurement and roundness measurement were evaluated to be $0.032 \mu \mathrm{m}$ and $0.024 \mu \mathrm{m}$, respectively.

Author Contributions: Conceptualization, W.G. and Y.S.; methodology, T.S., Q.L. and W.G.; software, T.S. and Q.L.; validation, Y.S., W.G. and H.M.; formal analysis, T.S., Q.L., Y.S. and W.G.; investigation, T.S., Q.L. and Y.S.; resources, Y.S. and W.G.; data curation, T.S., Y.S. and W.G; writing-original draft preparation, Q.L. and Y.S.; writing-review and editing, W.G. and Y.S.; visualization, W.G. and Y.S.; supervision, W.G.; project administration, W.G.; funding acquisition, W.G., Y.S. and H.M. All authors have read and agreed to the published version of the manuscript.

Funding: This work is supported by the Japan Society for the Promotion of Science (JSPS) 15H05759 and 20 H00211.

Acknowledgments: The authors would like to thank Yuki Machida for his help in the preparation of the experimental setup.

Conflicts of Interest: The authors declare no conflict of interest. The funders had no role in the design of the study; in the collection, analyses, or interpretation of data; in the writing of the manuscript, or in the decision to publish the results.

\section{References}

1. Uhlmann, E.; Mullany, B.; Biermann, D.; Rajurkar, K.P.; Hausotte, T.; Brinksmeier, E. Process chains for high-precision components with micro-scale features. CIRP Ann. 2016, 65, 549-572. [CrossRef]

2. ISO. ISO 1206 Rolling Bearings_-Needle Roller Bearings with Machined Rings_Boundary Dimensions, Geometrical Product specIfications (GPS) and Tolerance Values; ISO: Geneva, Switzerland, 2018.

3. Farhana, N.; Yusof, M.; Ripin, Z.M. Analysis of surface parameters and vibration of roller bearing. Tribol. Trans. 2014, 57, 715-729.

4. Haitjema, H.; Bosse, H.; Frennberg, M.; Sacconi, A.; Thalmann, R. International comparison of roundness profiles with nanometric accuracy. Metrologia 1996, 33, 67-73. [CrossRef]

5. Moore, W.R. Foundations of Mechanical Accuracy; Moore Special Tool Company, Inc.: Bridgeport, CT, USA, 1970.

6. Oberg, E.; Jones, F.D.; Horton, H.L.; Ryffel, H.H. Machinery's Handbook; Industrial Press, Inc.: New York, NY, USA, 2004. 
7. Shimizu, Y.; Gao, W.; Matsukuma, H.; Szipka, K.; Archenti, A. On-machine angle measurement of a precision V-groove on a ceramic workpiece. CIRP Ann. 2020, 00, 2-5. [CrossRef]

8. Talyrond 565/585HS, Taylor Hobson. Available online: www.taylor-hobson.com (accessed on 22 June 2020).

9. Weckenmann, A.; Bruning, J.; Patterson, S.; Knight, P. Grazing incidence interferometry for high precision measurements of cylindrical form deviations. CIRP Ann. 2001, 50, 381-384. [CrossRef]

10. Chen, Y.L.; Machida, Y.; Shimizu, Y.; Matsukuma, H.; Gao, W. A stitching linear-scan method for roundness measurement of small cylinders. CIRP Ann. 2018, 67, 535-538. [CrossRef]

11. Form Talysurf PGI, Taylor Hobson. Available online: www.taylor-hobson.com (accessed on 22 June 2020).

12. Gao, W.; Haitjema, H.; Fang, F.Z.; Leach, R.K.; Cheung, C.F.; Savio, E.; Linares, J.M. On-machine and in-process surface metrology for precision manufacturing. Cirp Ann. 2019, 68, 843-866. [CrossRef]

13. Joint Committee For Guides In Metrology. Evaluation of Measurement Data-Guide to the Expression of Uncertainty in Measurement; ISO: Geneva, Switzerland, 2008.

14. Takamasu, K. Least square method for precision measurement. J. Jpn. Soc. Precis. Eng. 2010, 76, 1130-1133. [CrossRef]

15. Helleseth, T. Some results about the cross-correlation function between two maximal linear sequences. Discret. Math. 1976, 16, 209-232. [CrossRef]

16. Helleseth, T. A note on the cross-correlation function between two binary maximal length linear sequences. Discret. Math. 1978, 23, 301-307. [CrossRef]

17. ISO 12181-1. Geometrical Product Specifications (GPS)-Roundness-Part 1: Vocabulary and Parameters of Roundness; ISO: Geneva, Switzerland, 2003.

18. Mitutoyo. Quick guide to precision measuring instruments. J. Prosthet. Dent. 2003, 11003, 2003.

19. MÖLLER-WEDEL OPTICAL Electronic Autocollimators. Available online: www.moeller-wedel-optical.com (accessed on 22 June 2020).

20. Gao, W.; Kim, S.W.; Bosse, H.; Haitjema, H.; Chen, Y.L.; Lu, X.D.; Knapp, W.; Weckenmann, A.; Estler, W.T.; Kunzmann, H. Measurement technologies for precision positioning. CIRP Ann. 2015, 64, 773-796. [CrossRef]

(C) 2020 by the authors. Licensee MDPI, Basel, Switzerland. This article is an open access article distributed under the terms and conditions of the Creative Commons Attribution (CC BY) license (http://creativecommons.org/licenses/by/4.0/). 